\title{
SOME WEIGHTED INTEGRAL INEQUALITIES FOR CONVEX FUNCTIONS
}

\author{
S.S. DRAGOMIR
}

ABstract. In this paper we establish some weighted integral inequalities of Čebyšev and Grüss' type for convex functions.

2010 Mathematics Subject Classification: 26D15; 25D10.

Keywords: Convex functions, Čebyšev's inequality, Grüss' inequality, Integral inequalities..

\section{INTRODUCTION}

For a function $f:[a, b] \rightarrow \mathbb{C}$ we consider the symmetrical transform of $f$ on the interval $[a, b]$, denoted by $\breve{f}_{[a, b]}$ or simply $\breve{f}$, when the interval $[a, b]$ is implicit, as defined by

$$
\breve{f}(t):=\frac{1}{2}[f(t)+f(a+b-t)], t \in[a, b] .
$$

For the Lebesgue measurable functions $q, h, k:[a, b] \rightarrow \mathbb{R}$ we introduce the weighted Čebyšev's functional

$$
\begin{aligned}
\mathcal{C}_{[a, b]}(h, k ; q):= & \frac{1}{\int_{a}^{b} q(t)} \int_{a}^{b} q(t) h(t) k(t) d t \\
& -\frac{1}{\int_{a}^{b} q(t)} \int_{a}^{b} q(t) h(t) d t \frac{1}{\int_{a}^{b} q(t)} \int_{a}^{b} q(t) k(t) d t=\mathcal{C}_{[a, b]}(k, h ; q)
\end{aligned}
$$

and the associated ( $\smile$ )- Čebyšev's functional

$$
\begin{aligned}
\breve{\mathcal{C}}_{[a, b]}(h, k ; q):= & \frac{1}{\int_{a}^{b} q(t)} \int_{a}^{b} \breve{q}(t) h(t) \breve{k}(t) d t \\
& -\frac{1}{\int_{a}^{b} q(t)} \int_{a}^{b} \breve{q}(t) h(t) d t \frac{1}{\int_{a}^{b} q(t)} \int_{a}^{b} \breve{q}(t) k(t) d t=\mathcal{C}_{[a, b]}(h, k ; \breve{q})
\end{aligned}
$$


provided that all the Lebesgue integrals exist on $[a, b]$ and $\int_{a}^{b} q(t) \neq 0$.

For $q \equiv 1$ we have the unweighted functionals

$$
\mathcal{C}_{[a, b]}(h, k):=\frac{1}{b-a} \int_{a}^{b} h(t) k(t) d t-\frac{1}{b-a} \int_{a}^{b} h(t) d t \frac{1}{b-a} \int_{a}^{b} k(t) d t
$$

and

$$
\breve{\mathcal{C}}_{[a, b]}(h, k):=\frac{1}{b-a} \int_{a}^{b} h(t) \breve{k}(t) d t-\frac{1}{b-a} \int_{a}^{b} h(t) d t \frac{1}{b-a} \int_{a}^{b} k(t) d t .
$$

It is well known that, if the functions $(h, k)$ are synchronous on $[a, b]$, namely

$$
[h(x)-h(y)][k(x)-k(y)] \geq 0
$$

for all $x, y \in[a, b]$ and $q$ is nonnegative, then the following weighted Čebyšev inequality holds

$$
0 \leq \breve{\mathcal{C}}_{[a, b]}(h, k ; q) .
$$

If there exists the constants $m, M, n, N$ such that $m \leq h \leq M$ and $n \leq k \leq N$ almost everywhere on $[a, b]$, then we also have the weighted Grüss inequality (see for instance [4] for an extension to general Lebesgue integral and positive measure):

$$
\breve{\mathcal{C}}_{[a, b]}(h, k ; q) \leq \frac{1}{4}(M-m)(N-n),
$$

with $\frac{1}{4}$ as best possible constant.

For other Čebyšev and Grüss' type inequalities see [1]-[9], [11]-[12] and [14]-[21].

In [13] the authors proved the following Čebyšev type inequality:

Theorem 1. Let $f, g:[a, b] \rightarrow \mathbb{R}$ be both convex or concave on $[a, b]$ and $p:[a, b] \rightarrow$ $[0, \infty)$ integrable an symmetric, namely $p(a+b-x)=p(x)$ for all $x \in[a, b]$, then

$$
\int_{a}^{b} p(x) d x \int_{a}^{b} p(x) f(x) \breve{g}(x) d x \geq \int_{a}^{b} p(x) f(x) d x \int_{a}^{b} p(x) g(x) d x .
$$

If one of the functions is convex and the other concave, then the sign of inequality reverses in (4).

If $p \equiv 1$ then for two functions that have the same convexity one can get from (4) that

$$
\begin{aligned}
\breve{\mathcal{C}}_{[a, b]}(f, g) & =\frac{1}{b-a} \int_{a}^{b} f(x) \breve{g}(x) d x-\frac{1}{b-a} \int_{a}^{b} f(x) d x \frac{1}{b-a} \int_{a}^{b} g(x) d x \\
& \geq 0 .
\end{aligned}
$$


Moreover, if in addition $g$ is symmetric then $\breve{g}(x)=g(x)$ and from (5) one obtains [13, Corollary 4]

$$
(b-a) \int_{a}^{b} f(x) g(x) d x \geq \int_{a}^{b} f(x) d x \int_{a}^{b} g(x) d x .
$$

In the subsequent paper [10] the authors provided an upper bound for $\breve{\mathcal{C}}_{[a, b]}(f, g)$ as follows:

Theorem 2. Let $f, g:[a, b] \rightarrow \mathbb{R}$ be both convex or concave on $[a, b]$, then

$$
\begin{aligned}
0 & \leq \breve{\mathcal{C}}_{[a, b]}(f, g) \\
& \leq \frac{1}{4}\left[\frac{f(a)+f(b)}{2}-f\left(\frac{a+b}{2}\right)\right]\left[\frac{g(a)+g(b)}{2}-g\left(\frac{a+b}{2}\right)\right],
\end{aligned}
$$

where the constant $\frac{1}{4}$ is best possible.

One can observe that for $g=f$ we get the inequality

$$
0 \leq \breve{\mathcal{C}}_{[a, b]}(f, f) \leq \frac{1}{4}\left[\frac{f(a)+f(b)}{2}-f\left(\frac{a+b}{2}\right)\right]^{2} .
$$

Motivated by the above results, in this paper we establish some new weighted integral inequalities of Čebyšev and Grüss' type for convex functions.

\section{Main Results}

We have the following fundamental relation between the Čebyšev's functional and the associated $(\smile)$-Čebyšev's functional:

Lemma 3. For the Lebesgue measurable functions $p, f, g:[a, b] \rightarrow \mathbb{R}$, we have the following equalities

$$
\mathcal{C}_{[a, b]}(\breve{f}, \breve{g} ; p)=\breve{\mathcal{C}}_{[a, b]}(f, g ; p)=\breve{\mathcal{C}}_{[a, b]}(g, f ; p),
$$

provided the involved Lebesgue integrals exist.

In particular

$$
\mathcal{C}_{[a, b]}(\breve{f}, \breve{g})=\breve{\mathcal{C}}_{[a, b]}(f, g)=\breve{\mathcal{C}}_{[a, b]}(g, f)
$$


Proof. We observe that

$$
\begin{aligned}
& \int_{a}^{b} p(t) \breve{f}(t) \breve{g}(t) d t \\
& =\frac{1}{4} \int_{a}^{b} p(t)[f(t)+f(a+b-t)][g(t)+g(a+b-t)] d t \\
& =\frac{1}{4}\left[\int_{a}^{b} p(t) f(t) g(t) d t+\int_{a}^{b} p(t) f(a+b-t) g(t) d t\right. \\
& \left.+\int_{a}^{b} p(t) f(t) g(a+b-t) d t+\int_{a}^{b} p(t) f(a+b-t) g(a+b-t) d t\right] .
\end{aligned}
$$

By using the change of variable $s=a+b-t, t \in[a, b]$ we have

$$
\int_{a}^{b} p(t) f(a+b-t) g(t) d t=\int_{a}^{b} p(a+b-t) f(t) g(a+b-t) d t
$$

and

$$
\int_{a}^{b} p(t) f(a+b-t) g(a+b-t) d t=\int_{a}^{b} p(a+b-t) f(t) g(t) d t .
$$

Therefore

$$
\int_{a}^{b} p(t) f(t) g(t) d t+\int_{a}^{b} p(a+b-t) f(t) g(t) d t=2 \int_{a}^{b} \breve{p}(t) f(t) g(t) d t
$$

and

$$
\begin{aligned}
& \int_{a}^{b} p(t) f(a+b-t) g(t) d t+\int_{a}^{b} p(t) f(t) g(a+b-t) d t \\
& =\int_{a}^{b} p(a+b-t) f(t) g(a+b-t) d t+\int_{a}^{b} p(t) f(t) g(a+b-t) d t \\
& =2 \int_{a}^{b} \breve{p}(t) f(t) g(a+b-t) d t .
\end{aligned}
$$

By using these identities we get

$$
\begin{aligned}
\int_{a}^{b} p(t) \breve{f}(t) \breve{g}(t) d t & =\frac{1}{4}\left[2 \int_{a}^{b} \breve{p}(t) f(t) g(t) d t+2 \int_{a}^{b} \breve{p}(t) f(t) g(a+b-t) d t\right] \\
& =\frac{1}{2}\left[\int_{a}^{b} \breve{p}(t) f(t) g(t) d t+\int_{a}^{b} \breve{p}(t) f(t) g(a+b-t) d t\right] \\
& =\int_{a}^{b} \breve{p}(t) f(t) \breve{g}(t) d t .
\end{aligned}
$$


Since

$$
\begin{aligned}
\int_{a}^{b} p(t) \breve{f}(t) d t & =\int_{a}^{b} p(t) \frac{f(t)+f(a+b-t)}{2} d t \\
& =\frac{1}{2}\left[\int_{a}^{b} p(t) f(t) d t+\int_{a}^{b} p(t) f(a+b-t) d t\right] \\
& =\frac{1}{2}\left[\int_{a}^{b} p(t) f(t) d t+\int_{a}^{b} p(a+b-t) f(t) d t\right]=\int_{a}^{b} \breve{p}(t) f(t) d t
\end{aligned}
$$

and, similarly

$$
\int_{a}^{b} p(t) \breve{g}(t) d t=\int_{a}^{b} \breve{p}(t) g(t) d t
$$

hence

$$
\begin{aligned}
& \int_{a}^{b} p(t) \int_{a}^{b} p(t) \breve{f}(t) \breve{g}(t) d t-\int_{a}^{b} p(t) \breve{f}(t) d t \int_{a}^{b} p(t) \breve{g}(t) d t \\
& =\int_{a}^{b} p(t) \int_{a}^{b} \breve{p}(t) f(t) \breve{g}(t) d t-\int_{a}^{b} \breve{p}(t) f(t) d t \int_{a}^{b} \breve{p}(t) g(t) d t
\end{aligned}
$$

which proves the first equality in (9).

The second equality follows by the symmetry of the Cebyšev functional.

Lemma 4 ([10, Lemma 2.2]). Let $f:[a, b] \rightarrow \mathbb{R}$ be convex (concave) on $[a, b]$, then $\breve{f}$ is nonincreasing (nondecreasing) on $\left[a, \frac{a+b}{2}\right]$ and nondecreasing (nonincreasing) on $\left[a, \frac{a+b}{2}\right]$.

We have the following weighted integral inequality:

Theorem 5. Assume that $f, g:[a, b] \rightarrow \mathbb{R}$ are both convex or concave on $[a, b]$ and $p:[a, b] \rightarrow[0, \infty)$ with $\int_{a}^{b} p(t) d t>0$. Then we have

$$
\begin{aligned}
0 \leq \breve{\mathcal{C}}_{[a, b]}(f, g ; p) & \\
\leq \frac{1}{4}\left[\frac{f(a)+f(b)}{2}\right. & \left.-f\left(\frac{a+b}{2}\right)\right]\left[\frac{g(a)+g(b)}{2}-g\left(\frac{a+b}{2}\right)\right] \\
& \leq \frac{1}{64}\left[f_{-}^{\prime}(b)-f_{+}^{\prime}(a)\right]\left[g_{-}^{\prime}(b)-g_{+}^{\prime}(a)\right](b-a)^{2}
\end{aligned}
$$

where

$$
\begin{aligned}
\breve{\mathcal{C}}_{[a, b]}(f, g ; p)=\frac{1}{\int_{a}^{b} p(t)} \int_{a}^{b} \breve{p}(t) & f(t) \breve{g}(t) d t \\
& \quad-\frac{1}{\int_{a}^{b} p(t)} \int_{a}^{b} \breve{p}(t) f(t) d t \frac{1}{\int_{a}^{b} p(t)} \int_{a}^{b} \breve{p}(t) g(t) d t .
\end{aligned}
$$


Proof. Since the set of differentiable convex functions defined on $(a, b)$ is dense in the class of all convex functions defined on $(a, b)$ in the uniform convergence topology, we can assume without loosing the generality that $f$ and $g$ are differentiable convex on $(a, b)$. This imply that $\breve{f}$ and $\breve{g}$ are differentiable convex and nonincreasing on $\left[a, \frac{a+b}{2}\right]$ and nondreasing on $\left[a, \frac{a+b}{2}\right]$.

For any $x, y \in[a, b]$ with $x \neq y$, by Cauchy mean value theorem, there exists a $c$ between $x$ and $y$ such that

$$
[\breve{f}(x)-\breve{f}(y)](\breve{g})^{\prime}(c)=[\breve{g}(x)-\breve{g}(y)](\breve{f})^{\prime}(c),
$$

which implies that

$$
[\breve{f}(x)-\breve{f}(y)][\breve{g}(x)-\breve{g}(y)](\breve{g})^{\prime}(c)=[\breve{g}(x)-\breve{g}(y)]^{2}(\breve{f})^{\prime}(c) .
$$

Since $\breve{f}$ and $\breve{g}$ are differentiable and nonincreasing on $\left[a, \frac{a+b}{2}\right]$ and nondreasing on $\left[a, \frac{a+b}{2}\right]$ then $(\breve{f})^{\prime}(c)$ and $(\breve{g})^{\prime}(c)$ have the same sign which implies that

$$
[\breve{f}(x)-\breve{f}(y)][\breve{g}(x)-\breve{g}(y)] \geq 0 .
$$

For $x=y$ the inequality (13) also holds, so $(\breve{f}, \breve{g})$ are synchronous on $[a, b]$.

Using the weighted Čebyšev's inequality (2) for $(\breve{f}, \breve{g})$ and $p$ we get

$$
0 \leq \mathcal{C}_{[a, b]}(\breve{f}, \breve{g} ; p)=\breve{\mathcal{C}}_{[a, b]}(f, g ; p) \text {, by Lemma } 3
$$

and the first inequality in (11) is proved.

Now, if we use the weighted Grüss' inequality (3) for the functions $\breve{f}, \breve{g}$ that satisfy the bounds

$$
f\left(\frac{a+b}{2}\right) \leq \breve{f} \leq \frac{f(a)+f(b)}{2}
$$

and

$$
g\left(\frac{a+b}{2}\right) \leq \breve{g} \leq \frac{g(a)+g(b)}{2}
$$

we get

$$
\begin{aligned}
& \mathcal{C}_{[a, b]}(\breve{f}, \breve{g} ; p) \\
& \leq \frac{1}{4}\left[\frac{f(a)+f(b)}{2}-f\left(\frac{a+b}{2}\right)\right]\left[\frac{g(a)+g(b)}{2}-g\left(\frac{a+b}{2}\right)\right]
\end{aligned}
$$


and by Lemma 3 we deduce the second inequality in (11).

In paper [8] we proved between others that for any convex function $h:[a, b] \rightarrow \mathbb{R}$ we have the inequality

$$
0 \leq \frac{h(a)+h(b)}{2}-h\left(\frac{a+b}{2}\right) \leq \frac{1}{4}\left[h_{-}^{\prime}(b)-h_{+}^{\prime}(a)\right](b-a)
$$

with the constant $\frac{1}{4}$ as best possible.

By using this inequality for $f$ and $g$ we deduce the last part of (11).

Remark 1. If $p$ is symmetrical on $[a, b]$, then by (11) we get

$$
\begin{aligned}
0 \leq \breve{\mathcal{C}}_{[a, b]}(f, g ; p)=\frac{1}{\int_{a}^{b} p(t)} \int_{a}^{b} p(t) f(t) \breve{g}(t) d t & \\
& \quad-\frac{1}{\int_{a}^{b} p(t)} \int_{a}^{b} p(t) f(t) d t \frac{1}{\int_{a}^{b} p(t)} \int_{a}^{b} p(t) g(t) d t
\end{aligned}
$$

and therefore we recapture the inequality (5).

The second inequality in (11) gives a weighted generalization of the inequality (7).

Corollary 6. Assume that $f:[a, b] \rightarrow \mathbb{R}$ is either convex or concave on $[a, b]$ and $p:[a, b] \rightarrow[0, \infty)$ with $\int_{a}^{b} p(t) d t>0$. Then we have

$$
0 \leq \breve{\mathcal{C}}_{[a, b]}(f, f ; p) \leq \frac{1}{4}\left[\frac{f(a)+f(b)}{2}-f\left(\frac{a+b}{2}\right)\right]^{2} \leq \frac{1}{64}\left[f_{-}^{\prime}(b)-f_{+}^{\prime}(a)\right]^{2}
$$

where

$$
\breve{\mathcal{C}}_{[a, b]}(f, f ; p)=\frac{1}{\int_{a}^{b} p(t)} \int_{a}^{b} \breve{p}(t) f(t) \breve{f}(t) d t-\left(\frac{1}{\int_{a}^{b} p(t)} \int_{a}^{b} \breve{p}(t) f(t) d t\right)^{2} .
$$

\section{Related Results}

We can improve the second inequality in (11) as follows: 
Theorem 7. Assume that $f, g:[a, b] \rightarrow \mathbb{R}$ are both convex on $[a, b]$ and $p:[a, b] \rightarrow$ $[0, \infty)$ with $\int_{a}^{b} p(t) d t>0$.

$$
\begin{aligned}
0 \leq \breve{\mathcal{C}}_{[a, b]}(f, g ; p) & \leq\left(\frac{f(a)+f(b)}{2}-\frac{1}{\int_{a}^{b} p(t) d t} \int_{a}^{b} \breve{p}(t) f(t) d t\right)^{1 / 2} \\
& \times\left(\frac{1}{\int_{a}^{b} p(t) d t} \int_{a}^{b} \breve{p}(t) f(t) d t-f\left(\frac{a+b}{2}\right)\right)^{1 / 2} \\
& \times\left(\frac{g(a)+g(b)}{2}-\frac{1}{\int_{a}^{b} p(t) d t} \int_{a}^{b} \breve{p}(t) g(t) d t\right)^{1 / 2} \\
& \times\left(\frac{1}{\int_{a}^{b} p(t) d t} \int_{a}^{b} \breve{p}(t) g(t) d t-g\left(\frac{a+b}{2}\right)\right)^{1 / 2} \\
\leq \frac{1}{4}\left[\frac{f(a)+f(b)}{2}-f\left(\frac{a+b}{2}\right)\right]\left[\frac{g(a)+g(b)}{2}-g\left(\frac{a+b}{2}\right)\right] & \frac{1}{64}\left[f_{-}^{\prime}(b)-f_{+}^{\prime}(a)\right]\left[g_{-}^{\prime}(b)-g_{+}^{\prime}(a)\right](b-a)^{2} .
\end{aligned}
$$

Proof. We employ the following well known inequality that follows from the weighted Korkine's identity [15, p. 296] and the weighted double integral Cauchy-BunyakovskySchwarz inequality

$$
\left[\mathcal{C}_{[a, b]}(h, k ; q)\right]^{2} \leq\left[\mathcal{C}_{[a, b]}(h, h ; q)\right]\left[\mathcal{C}_{[a, b]}(k, k ; q)\right]
$$

see also [5].

We also have the identity [5, p. 399]

$$
\begin{aligned}
& \mathcal{C}_{[a, b]}(h, h ; q) \\
& =\left(\Phi-\frac{1}{\int_{a}^{b} q(t) d t} \int_{a}^{b} q(t) h(t) d t\right)\left(\frac{1}{\int_{a}^{b} q(t)} \int_{a}^{b} q(t) h(t) d t-\phi\right) \\
& -\frac{1}{\int_{a}^{b} q(t) d t} \int_{a}^{b} q(t)[\Phi-h(t)][h(t)-\phi] d t
\end{aligned}
$$

and if $[\Phi-h(t)][h(t)-\phi] \geq 0$ and $q(t) \geq 0$ for $t \in[a, b]$, then

$$
\begin{array}{r}
\mathcal{C}_{[a, b]}(h, h ; q) \leq \\
\left(\Phi-\frac{1}{\int_{a}^{b} q(t) d t} \int_{a}^{b} q(t) h(t) d t\right)\left(\frac{1}{\int_{a}^{b} q(t) d t} \int_{a}^{b} q(t) h(t) d t-\phi\right) \\
\leq \frac{1}{4}(\Phi-\phi)^{2} .
\end{array}
$$


From (18) we have

$$
\left[\mathcal{C}_{[a, b]}(\breve{f}, \breve{g} ; p)\right]^{2} \leq\left[\mathcal{C}_{[a, b]}(\breve{f}, \breve{f} ; p)\right]\left[\mathcal{C}_{[a, b]}(\breve{g}, \breve{g} ; p)\right]
$$

and since $\breve{f}$ and $\breve{g}$ satisfy the conditions (14) and (15), hence

$$
\begin{aligned}
\mathcal{C}_{[a, b]}(\breve{f}, \breve{f} ; p) & \leq\left(\frac{f(a)+f(b)}{2}-\frac{1}{\int_{a}^{b} p(t) d t} \int_{a}^{b} p(t) \breve{f}(t) d t\right) \\
& \times\left(\frac{1}{\int_{a}^{b} p(t) d t} \int_{a}^{b} p(t) \breve{f}(t) d t-f\left(\frac{a+b}{2}\right)\right) \\
& \leq \frac{1}{4}\left[\frac{f(a)+f(b)}{2}-f\left(\frac{a+b}{2}\right)\right]^{2}
\end{aligned}
$$

and

$$
\begin{aligned}
\mathcal{C}_{[a, b]}(\breve{g}, \breve{g} ; p) \leq & \left(\frac{g(a)+g(b)}{2}-\frac{1}{\int_{a}^{b} p(t) d t} \int_{a}^{b} p(t) \breve{g}(t) d t\right) \\
& \times\left(\frac{1}{\int_{a}^{b} p(t) d t} \int_{a}^{b} p(t) \breve{g}(t) d t-g\left(\frac{a+b}{2}\right)\right) \\
\leq & \frac{1}{4}\left[\frac{g(a)+g(b)}{2}-g\left(\frac{a+b}{2}\right)\right]^{2} .
\end{aligned}
$$

By taking into account that

$$
\int_{a}^{b} p(t) \breve{f}(t) d t=\int_{a}^{b} \breve{p}(t) f(t) d t \text { and } \int_{a}^{b} p(t) \breve{g}(t) d t=\int_{a}^{b} \breve{p}(t) g(t) d t
$$

and by making use of (21), (22) and the representation Lemma 3 we deduce the desired result (17). 
Remark 2. If $p$ is symmetrical on $[a, b]$, then by (17) we get

$$
\begin{aligned}
0 \leq \breve{\mathcal{C}}_{[a, b]}(f, g ; p) & \leq\left(\frac{f(a)+f(b)}{2}-\frac{1}{\int_{a}^{b} p(t) d t} \int_{a}^{b} p(t) f(t) d t\right)^{1 / 2} \\
& \times\left(\frac{1}{\int_{a}^{b} p(t) d t} \int_{a}^{b} p(t) f(t) d t-f\left(\frac{a+b}{2}\right)\right)^{1 / 2} \\
& \times\left(\frac{g(a)+g(b)}{2}-\frac{1}{\int_{a}^{b} p(t) d t} \int_{a}^{b} p(t) g(t) d t\right)^{1 / 2} \\
& \times\left(\frac{1}{\int_{a}^{b} p(t) d t} \int_{a}^{b} p(t) g(t) d t-g\left(\frac{a+b}{2}\right)\right)^{1 / 2} \\
\leq \frac{1}{4}\left[\frac{f(a)+f(b)}{2}-f\left(\frac{a+b}{2}\right)\right]\left[\frac{g(a)+g(b)}{2}-g\left(\frac{a+b}{2}\right)\right] & \frac{1}{64}\left[f_{-}^{\prime}(b)-f_{+}^{\prime}(a)\right]\left[g_{-}^{\prime}(b)-g_{+}^{\prime}(a)\right](b-a)^{2} .
\end{aligned}
$$

Corollary 8. Assume that $f:[a, b] \rightarrow \mathbb{R}$ is either convex or concave on $[a, b]$ and $p:[a, b] \rightarrow[0, \infty)$ with $\int_{a}^{b} p(t) d t>0$. Then we have

$$
\begin{aligned}
0 \leq \breve{\mathcal{C}}_{[a, b]}(f, f ; p) & \\
\leq & \left(\frac{f(a)+f(b)}{2}-\frac{1}{\int_{a}^{b} p(t) d t} \int_{a}^{b} \breve{p}(t) f(t) d t\right) \\
& \times\left(\frac{1}{\int_{a}^{b} p(t) d t} \int_{a}^{b} \breve{p}(t) f(t) d t-f\left(\frac{a+b}{2}\right)\right) \\
& \leq \frac{1}{4}\left[\frac{f(a)+f(b)}{2}-f\left(\frac{a+b}{2}\right)\right]^{2} \leq \frac{1}{64}\left[f_{-}^{\prime}(b)-f_{+}^{\prime}(a)\right]^{2} .
\end{aligned}
$$

The following case for unweighted inequalities is of interest: 
Corollary 9. Let $f, g:[a, b] \rightarrow \mathbb{R}$ be both convex on $[a, b]$, then

$$
\begin{aligned}
0 \leq \breve{\mathcal{C}}_{[a, b]}(f, g) \leq\left(\frac{f(a)+f(b)}{2}-\frac{1}{b-a} \int_{a}^{b} f(t) d t\right)^{1 / 2} \\
\quad \times\left(\frac{1}{b-a} \int_{a}^{b} f(t) d t-f\left(\frac{a+b}{2}\right)\right)^{1 / 2} \\
\times\left(\frac{g(a)+g(b)}{2}-\frac{1}{b-a} \int_{a}^{b} g(t) d t\right)^{1 / 2} \\
\times\left(\frac{1}{b-a} \int_{a}^{b} g(t) d t-g\left(\frac{a+b}{2}\right)\right)^{1 / 2} \\
\leq \frac{1}{4}\left[\frac{f(a)+f(b)}{2}-f\left(\frac{a+b}{2}\right)\right]\left[\frac{g(a)+g(b)}{2}-g\left(\frac{a+b}{2}\right)\right] \\
\leq \frac{1}{64}\left[f_{-}^{\prime}(b)-f_{+}^{\prime}(a)\right]\left[g_{-}^{\prime}(b)-g_{+}^{\prime}(a)\right](b-a)^{2} .
\end{aligned}
$$

\section{REFERENCES}

[1] A. Aglić Aljinović, R. Hoxha and J. Pečarić, On some Čebyšev-Grüss type integral inequalities. Mat. Bilten No. 30 (2006), 49-62

[2] P. R. Beesack and J. E. Pečarić, Integral inequalities of Čebyšev type. J. Math. Anal. Appl. 111 (1985), no. 2, 643-659.

[3] K. Boukerrioua and A. Guezane-Lakoud, On generalization of Čebyšev type inequalities. J. Inequal. Pure Appl. Math. 8 (2007), No. 2, Article 55, 4 pp.

[4] P. Cerone and S. S. Dragomir, A refinement of the Grüss inequality and applications, Tamkang J. Math. 38 (2007), no. 1, 37-49,

Preprint RGMIA Res. Rep. Coll. 5 (2002), No. 2, Art. 14. [Online https://rgmia.org/v5n2.php] .

[5] S. S. Dragomir, Some integral inequalities of Grüss type. Indian J. Pure Appl. Math. 31 (2000), no. 4, 397-415.

[6] S. S. Dragomir, On some improvements of Čebyšev's inequality for sequences and integrals. Studia Univ. Babeş-Bolyai Math. 35 (1990), no. 4, 35-40.

[7] S. S. Dragomir, A refinement of Ostrowski's inequality for the Čebyšev functional and applications. Analysis (Munich) 23 (2003), No. 4, 287-297.

[8] S. S. Dragomir, Bounds for the deviation of a function from the chord generated by its extremities. Bull. Aust. Math. Soc. 78 (2008), no. 2, 225-248. 
S.S. Dragomir - Integral Inequalities for Convex Functions ...

[9] S. S. Dragomir and B. Mond, Some mappings associated with Cebysev's inequality for sequences of real numbers. Bull. Allahabad Math. Soc. 8/9 (1993/94), 37-55 (1997).

[10] A. El Farissi and Z. Latreuch, New type of Chebychev-Grüss inequalities for convex functions, Acta Univ. Apulensis Math. Inform. No. 34 (2013), 235-245.

[11] A Guezane-Lakoud and F. Aissaoui, New Čebyšev type inequalities for double integrals. J. Math. Inequal. 5 (2011), No. 4, 453-462.

[12] S.-R. Hwang, Ostrowski-Grüss-Čebyšev type inequalities involving several functions. Tamsui Oxf. J. Math. Sci. 23 (2007), No. 1, 105-125.

[13] Z. Latreuch, and B. Belaïdi, Like Chebyshev's inequalities for convex functions and applications. Acta Univ. Apulensis Math. Inform. No. 35 (2013), 83-94.

[14] Z. Liu, Generalizations of some new Čebyšev type inequalities. J. Inequal. Pure Appl. Math. 8 (2007), No. 1, Article 13, 6 pp.

[15] D. S. Mitrinović, J. E. Pečarić and A. M. Fink, Classical and New Inequalities in Analysis. Mathematics and its Applications (East European Series), 61. Kluwer Academic Publishers Group, Dordrecht, 1993. xviii+740 pp. ISBN: 0-7923-2064-6

[16] B. G. Pachpatte, On Ostrowski-Grüss-Čebyšev type inequalities for functions whose modulus of derivatives are convex. J. Inequal. Pure Appl. Math. 6 (2005), No. 4, Article 128, 15 pp.

[17] B. G. Pachpatte, New inequalities of Čebyšev type for double integrals, Demonstratio Math. 40 (2007), no. 1, 43-50.

[18] B. G. Pachpatte, A note on Čebyšev type inequalities. An. Ştiinţ. Univ. Al. I. Cuza Iaşi. Mat. (N.S.) 53 (2007), no. 1, 97-102.

[19] R. T. Rahmail, Inversion of Čebyšev's inequality for bounded functions. (Russian) Izv. Vyš̌. Učebn. Zaved. Matematika 1977, No. 12 (187), 77-84.

[20] E. Set, M. Z. Sarikaya and F. Ahmad, A generalization of Čebyšev type inequalities for first differentiable mappings. Miskolc Math. Notes 12 (2011), No. 2, $245-253$.

[21] F. Zafar and N. A. Mir, A note on the generalization of some new Čebyšev type inequalities. Tamsui Oxf. J. Inf. Math. Sci. 27 (2011), No. 2, 149-157.

Silvestru Sever Dragomir

College of Engineering and Science,

Victoria University,

Melbourne, Australia

email: sever.dragomir@vu.edu.au 